Pure and Applied Mathematics Quarterly

Volume 7, Number 2

(Special Issue: In honor of

Frederick W. Gehring, Part 2 of 2$)$

$455-468,2011$

\title{
Teichmüller Mappings, Quasiconformal Homogeneity, and Non-amenable Covers of Riemann Surfaces
}

Petra Bonfert-Taylor, Gaven Martin, Alan W. Reid and Edward C. Taylor

To F.W. Gehring - friend, mentor, and inadvertent match-maker*

\begin{abstract}
We show that there exists a universal constant $K_{c}$ so that every $K$-strongly quasiconformally homogeneous hyperbolic surface $X$ (not equal to $\mathbb{H}^{2}$ ) has the property that $K>K_{c}>1$. The constant $K_{c}$ is the best possible, and is computed in terms of the diameter of the $(2,3,7)$-hyperbolic orbifold (which is the hyperbolic orbifold of smallest area.) We further show that the minimum strong homogeneity constant of a hyperbolic surface without conformal automorphisms decreases if one passes to a non-amenable regular cover.
\end{abstract}

Keywords: Quasiconformal homogeneity, Riemann surface, hyperbolic orbifold.

\section{Introduction and Statement of Results}

Recall that an orientable hyperbolic $n$-manifold $N$ is uniformly quasiconformally homogeneous if there exists a constant $K \geq 1$ so that for any two points $x, y \in N$ there exists a $K$-quasiconformal automorphism of $N$ that pairs $x$ and

Received March 27, 2007.

The first and fourth authors were supported in part by NSF grant DMS-0305704.

The second author was supported in part by the Marsden Fund, NZ.

The third author was supported in part by NSF grant DMS-0503753. This author would also like to thank Wesleyan University for its hospitality during this work.

*Two of the authors met and married while being postdoctoral students at the University of Michigan. Fred Gehring was instrumental in the events that made their meeting possible. 
$y$. The concept of quasiconformal homogeneity was introduced and developed by Gehring and Palka in [7]; for other work on quasiconformally homogeneous structures see [8], [9], [4], and [5]. In dimensions three and above, owing to well-known quasiconformal rigidity phenomena, the property of being uniformally quasiconformally homogeneous is a topologically restrictive one, we recall Theorem 1.3 of [4].

Theorem 1.1. Let $N$ be an orientable hyperbolic $n$-manifold, with $n \geq 3$. Then $N$ is uniformly quasiconformally homogeneous if and only if $N$ is the regular cover of a closed hyperbolic orbifold.

Because quasiconformal rigidity phenomena fail in dimension two such a strong topological classification is unlikely to be true for surfaces. However by strengthening the definition of quasiconformal homogeneity, one can construct a setting from which interesting questions can be posed concerning the analytic properties of quasiconformal automorphisms of surfaces. A hyperbolic surface (equivalently a Riemann surface) $X$ is strongly quasiconformally homogeneous ([5]) if there exists a constant $K \geq 1$ so that for any two points $x, y \in X$ there is a $K$ quasiconformal automorphism $f: X \rightarrow X$ so that $y=f(x)$ and so that $f$ is homotopic to a conformal automorphism $c: X \rightarrow X$; we also say that $X$ is $K$ strongly quasiconformally homogeneous. Using results of Gehring and Palka, it is elementary to see that every closed hyperbolic surface is strongly quasiconformally homogeneous, and thus this definition applies to a broad class of hyperbolic surfaces.

We can provide a complete classification of strongly quasiconformally homogeneous surfaces, and in fact this is what motivates our interest in them. Using the argument used to prove Theorem 1.1, we observe:

Theorem 1.2. Let $X$ be an orientable hyperbolic surface. Then $X$ is strongly quasiconformally homogeneous if and only if $X$ is a regular cover of a closed hyperbolic orbifold.

See the proof of Theorem 1.3 in [4]. Of course, there are many covers of a closed hyperbolic surface which are not regular covers and it is thus easy to construct an example of a hyperbolic surface $X$ on which the injectivity radius function is bounded between two constants, and yet $X$ is not uniformly quasiconformally homogeneous. 
Suppose that $X$ is strongly quasiconformally homogeneous, and let

$K_{\text {aut }}(X)=\inf \{K \mid X$ is $K$-strongly quasiconformally homogeneous $\}$.

Using a normal family argument it is not hard to show that a strongly quasiconformally homogeneous hyperbolic surface $X$ is in fact $K_{\text {aut }}(X)$-strongly quasiconformally homogeneous. We define

$K_{c}=\inf \left\{K_{\text {aut }}(X) \mid X \neq \mathbb{H}^{2}\right.$ is strongly quasiconformally homogeneous $\}$.

One of the main results in this note is:

Theorem 1.3. The constant $K_{c}$ is strictly greater than one, and can be calculated in terms of the diameter of the quotient of $\mathbb{H}^{2}$ by the $(2,3,7)$-group. Furthermore no strongly quasiconformally homogeneous hyperbolic surface $X \neq \mathbb{H}^{2}$ is $K_{c^{-}}$ strongly quasiconformally homogeneous, that is, $K_{\text {aut }}(X)$ is strictly greater than $K_{c}$.

\section{Remarks:}

(1) Each uniformly quasiconformally homogeneous hyperbolic surface $X$ that is not $\mathbb{H}^{2}$ has the property that $K(X)>1$. (See Proposition 2.2 of [4].) This follows from the fact that a 1-quasiconformal mapping is conformal, and from the fact that the conformal automorphism group of a non-elementary hyperbolic surface acts discontinuously on the surface.

(2) Theorem 1.3 is in fact a refinement of a result of Bonfert-Taylor, Bridgeman, Canary and Taylor; see Theorem 6.5 of [5]. The refinement here is that we provide a sharp lower bound on the strong homogeneity constant $K_{c}$.

In the proof of the following lemma we will need to localize our analysis. For a strongly quasiconformally homogeneous hyperbolic surface $X$ we define the function

$$
K_{\text {aut }}(x, y)=\min _{f}\{K(f)\}
$$

where the minimum is found over all quasiconformal mappings $f: X \rightarrow X$ which are homotopic to a conformal automorphism and for which $y=f(x)$. That the infimum is achieved is an elementary consequence of compactness properties of quasiconformal mappings. 
Now let $X$ be a closed hyperbolic surface having trivial conformal automorphism group, and let $Y$ be a regular cover of $X$. Though $X$ has trivial automorphism group it remains strongly quasiconformally homogeneous since it is compact. We now establish the following useful lemma.

Lemma 1.4. Let $X$ be a closed hyperbolic surface having trivial automorphism group, and let $Y$ be any regular cover of $X$. Then $K_{\text {aut }}(Y) \leq K_{\text {aut }}(X)$.

Proof. Because the surface $X$ has trivial automorphism group, for each pair of points $x_{1}, x_{2} \in X$ a best mapping realizing $K_{a u t}\left(x_{1}, x_{2}\right)$ is homotopic to the identity and thus lifts to a family of $K_{\text {aut }}\left(x_{1}, x_{2}\right)$-quasiconformal automorphisms of $Y$, each homotopic to a conformal automorphism of $Y$, and so that for each such mapping there exist a pair of points $y_{1}, y_{2}$ (respectively) in the fibers $\pi^{-1}\left(x_{1}\right) \in$ $Y$ and $\pi^{-1}\left(x_{2}\right) \in Y$ that is paired by the mapping. Thus one observes that $K_{\text {aut }}\left(y_{1}, y_{2}\right) \leq K_{\text {aut }}\left(x_{1}, x_{2}\right)$ for all $y_{1} \in \pi^{-1}\left(x_{1}\right)$ and $y_{2} \in \pi^{-1}\left(x_{2}\right)$. Because $Y$ covers $X$ regularly the result follows.

Remark. It is well known (e.g. see [6], section 3.2) that for genus $g \geq 3$ the set of closed surfaces in Teichmüller space having only trivial automorphism group is of full measure.

We show in [4] that if $M^{n}$ is a uniformly quasiconformally homogeneous hyperbolic manifold of any dimension $n \geq 2$, then $K\left(M^{n}\right)>1$ if and only if $M^{n} \neq \mathbb{H}^{n}$; note that $\mathbb{H}^{n}$ is a non-amenable cover of any hyperbolic manifold with non-elementary fundamental group. In the setting of closed hyperbolic surfaces having trivial automorphism group we show that passing to any non-amenable regular cover strictly decreases $K_{\text {aut }}$ :

Theorem 1.5. Let $X$ be a closed hyperbolic surface having trivial automorphism group, and let $Y$ be a non-amenable regular cover of $X$. Then $K_{\text {aut }}(Y)<$ $K_{\text {aut }}(X)$.

\section{The Proof of Theorem 1.3}

In this section we will prove Theorem 1.3. Before doing so, we will need to recall some basic definitions and facts. First, in the definition of strong quasiconformal homogeneity we are assuming that each allowable quasiconformal 
mapping is homotopic to a conformal mapping. Thus we can convert each allowable quasiconformal mapping, via post-composition with the inverse of the conformal automorphism, into one which is homotopic to the identity having the same dilatation. Since we wish to measure the size of the dilation the following function will be of use. Let $\psi:[0, \infty) \rightarrow[1, \infty)$ be the function which gives the best dilatation over all quasiconformal homeomorphisms of $\mathbb{D}^{2}$ that are homotopic to the identity and move the origin 0 a prescribed distance $d \in[0, \infty)$, i.e.

$\psi(d)=\min \left\{K \geq 1 \mid\right.$ there exists $h: \mathbb{D}^{2} \rightarrow \mathbb{D}^{2}, K-q c, h \mid \partial \mathbb{D}^{2}=$ id, $\left.\rho(0, h(0))=d\right\}$.

(Here, of course, $\left(\mathbb{D}^{2}, \rho\right)$ denotes the ball model of 2-dimensional hyperbolic space of constant curvature -1 .) We record the following explicit formula for $\psi$, due originally to Teichmüller [13].

Proposition 2.1. Let $f: \mathbb{D}^{2} \rightarrow \mathbb{D}^{2}$ be a quasiconformal map which extends to the identity on the unit circle. Then $K(f) \geq \psi(\rho(0, f(0)))$, where $\psi:[0, \infty) \rightarrow[1, \infty)$ is the increasing homeomorphism given by the function

$$
\psi(d)=\operatorname{coth}^{2}\left(\frac{\pi^{2}}{4 \mu\left(e^{-d}\right)}\right)=\operatorname{coth}^{2} \mu\left(\sqrt{1-e^{-2 d}}\right),
$$

and $\mu(r)$ is the modulus of the Grötsch ring whose complementary components are $\overline{\mathbb{D}^{2}}$ and $[1 / r, \infty]$ for $0<r<1$. In particular,

$$
\psi(d) \sim \frac{16 d^{2}}{\pi^{4}} \text { as } d \rightarrow \infty \quad \text { and } \quad \psi(d) \sim 1+\frac{d}{2} \text { as } d \rightarrow 0 .
$$

The critical value of $d$, for our analysis, is the minimum diameter of a hyperbolic orbifold (surface). In fact, the minimum diameter hyperbolic orbifold in dimension two is the minimum volume hyperbolic orbifold, that is, the orbifold built by the $(2,3,7)$-triangle group. The following fact is known, however we include a proof for convenience.

Proposition 2.2. The minimum diameter hyperbolic orbifold $O_{\min }$ is the $(2,3,7)$ hyperbolic orbifold.

Proof. Recall the isodiametric inequality in hyperbolic 2-space: If a planar set has diameter $d(d>0)$ then the area of the planar set is less than or equal to $4 \pi \sinh ^{2}\left(\frac{d}{4}\right)$ (e.g. see [12], also recall that $4 \pi \sinh ^{2}\left(\frac{d}{4}\right)$ is the area of a hyperbolic disk of radius $\frac{d}{2}$.) Using the convex polyhedron (say a Dirichlet polyhedron) of a 
closed orbifold $O^{2}$ of diameter $d<\infty$, we easily see that the hyperbolic area of $O^{2}$ is thus less than or equal to $4 \pi \sinh ^{2}\left(\frac{d}{4}\right)$.

We now consider the $(2,3,7)$-triangle group. It is an easy exercise in hyperbolic trigonometry (e.g. see [2]) that the diameter of the orbifold quotient of $\mathbb{H}^{2}$ by the $(2,3,7)$-triangle group is approximately 0.62067 . Using this value for $d$, we observe from the isodiametric inequality that any orbifold having diameter less than the diameter of the $(2,3,7)$-triangle orbifold must have area less than 0.305 .

Using this area bound we can now systematically rule out the possibility that whole classes of Fuchsian groups have quotient orbifolds of diameter less than 0.62067. First note that it is clear that any Fuchsian group that contains a parabolic, or is of the second kind, or has infinitely generated fundamental group, has a quotient surface of infinite diameter and thus is not a candidate. From the area signature formula (see Theorem 10.4.3 in [2]), we immediately observe that if the genus of any such orbifold is greater than or equal to 1 then its area is greater than or equal to $\pi$ and thus is too large to have diameter less than 0.62067. In fact, any admissable Fuchsian group of genus 0 and with signature $\left(0: m_{1}, \ldots, m_{r}\right)$ with $r \geq 4$ will have area that is strictly greater than 0.305 , and so these groups are ruled out as well.

Thus we are left to consider groups of signature $\left(0: m_{1}, m_{2}, m_{3}\right)$, where without loss of generality we can assume $m_{1} \leq m_{2} \leq m_{3}$. The basic idea is to observe that there is a monotonicity in the size of diameter in terms of the values of $m_{1}, m_{2}$ and $m_{3}$. Using hyperbolic trigonometry one can first explicitly show that if $m_{1}=2$, then the diameter of the quotient of any admissable Fuchsian group of that signature is strictly greater than 0.62067 . Now one considers admissible signatures for which $m_{1} \geq 3$. Once again, by explicit calculation using this monotonicity, we need only check a finite number of signatures and so we are able to rule these out as well.

Remark. We conjecture that a minimum diameter hyperbolic 3-orbifold is the orientation-preserving half of the $\mathbb{Z}_{2}$-extension of the Coxeter 3-5-3 reflection group.

We restate Theorem 1.3 in terms of the discussion above.

Theorem 2.3. We have that $K_{c}=\psi\left(\right.$ diam $\left.O_{\text {min }}\right) \approx 1.36138$. Furthermore, any strongly quasiconformally homogeneous surfaces $R \neq \mathbb{H}^{2}$ satisfies $K_{\text {aut }}(R)>K_{c}$. 
Proof. We will first construct a sequence $\left\{S_{n}\right\}$ of surfaces such that the infimum over the sequence has the property that $\inf K_{a u t}\left(S_{n}\right) \leq \psi\left(\operatorname{diam} O_{\min }\right)$. Then we will show that $K_{\text {aut }}(S)>\psi\left(\right.$ diam $\left.O_{\min }\right)$ for each surface $S$, and this will complete the proof of the theorem.

By a standard geometric application of residual finiteness of surface groups (see [11]) we can construct a sequence of regular closed hyperbolic surface covers $\left\{S_{n}\right\}$ of $O_{\text {min }}$, such that their minimal injectivity radii $\ell\left(S_{n}\right)$ go to infinity as $n \rightarrow \infty$.

Let $x, y \in S_{n}$, then there exists $g \in \operatorname{Aut}\left(S_{n}\right)$ such that $\rho(g(x), y) \leq \operatorname{diam} O_{\text {min }}$. By construction the injectivity radius at the point $g(x)$ is necessarily large for all large index $n$ independent of the choice of $x$ and $y$. Thus we have that there exists a quasiconformal homeomorphism $f: S_{n} \rightarrow S_{n}$ satisfying the following conditions: first, $f=$ id outside of $B\left(g(x), i n j_{g(x)}\right)$ and $f(g(x))=y$, and secondly

$$
K(f) \leq \psi\left(\operatorname{diam} O_{\min }\right)+\varepsilon_{n},
$$

where $\varepsilon_{n}$ is independent of $x$ and $y$ and gets smaller as the injectivity radius gets larger and thus in the limit $\varepsilon_{n} \rightarrow 0$ as $n \rightarrow \infty$. To verify this, observe that the hyperbolic distance between $g(x)$ and $y$ in the hyperbolic metric of the disk $B\left(g(x), i n j_{g(x)}\right)$ is only slightly larger than it is in $S_{n}$ if $i n j_{g(x)}$ is large enough. We can thus transport Teichmüller's extremal map into this disk and use the identity map outside of the disk to map $g(x)$ to $y$. Thus

$$
\inf \left\{K_{\text {aut }}\left(S_{n}\right) \mid n \in \mathbb{N}\right\} \leq \psi\left(\operatorname{diam} O_{\text {min }}\right) .
$$

Next we show that any strongly quasiconformally homogeneous surface $S \neq \mathbb{H}^{2}$ satisfies that $K_{\text {aut }}(S)>K_{c}$.

Let $S \neq \mathbb{H}^{2}$ be an arbitrary strongly quasiconformally homogeneous surface, and choose $x, y \in S$ such that

$$
\min \{\rho(x, g(y)) \mid g \in \operatorname{Aut}(S)\} \geq \operatorname{diam} O_{\text {min }} .
$$

By composing with conformal automorphisms we can furthermore assume that a least dilatation mapping $f$ that maps $x$ to $y$ while being homotopic to a conformal automorphism is in fact homotopic to the identity. We will show that $K(f)>K_{c}$ and this shows that $K_{\text {aut }}(S)>K_{c}$.

Let $p: \mathbb{D}^{2} \rightarrow S$ be a universal covering map such that $p(0)=x$ and $p(-\sigma)=y$, where $\rho(0,-\sigma)=\rho(x, y)$ (and $\sigma \in(0,1)$. Let $\tilde{f}$ be a lift of $f$ to the unit disk such 
that $\tilde{f}(0)=-\sigma$. Then the extension of $\tilde{f}$ to the unit circle is the identity map of the unit circle. Hence, using Proposition 2.1, we have that $K(\tilde{f}) \geq \psi(\rho(0,-\sigma)) \geq$ $\psi\left(\operatorname{diam}\left(O_{\min }\right)\right)$. We will show that the first of these two inequalities is strict.

First note that $\tilde{f}$ cannot be Teichmüller's unique minimal map (i.e. the unique minimum dilatation quasiconformal mapping $\Phi$ of the unit disk to itself that extends to the identity on the boundary and maps the origin to the point $-\sigma$ ). The key idea here is that Teichmüller's extremal map cannot be compatible with any Fuchsian group of the first kind, and thus cannot live on any strongly quasiconformally homogeneous surface (compare Theorem 1.2).

A general argument can be made using the fact that the unit disk is a nonamenable cover of the surface $S$ (see Lemma 3.2), but we can give an explicit argument which only requires a geometric understanding of Teichmüller's minimal $\operatorname{map} \Phi([13])$.

Let $O$ be the double cover of the unit disk, branched at the origin. Let $\phi_{1}$ : $O \rightarrow \mathbb{D}^{2}$ be the function $\phi_{1}(z)=\sqrt{z}$ which maps $O \backslash\{0\}$ conformally onto the unit disk minus the origin. The two slits from 0 to $-\sigma$ on the two leafs of $O$ get mapped onto the line segment $[-i \sqrt{\sigma}, i \sqrt{\sigma}]$. The unit disk minus this slit can be mapped conformally by a mapping $\phi_{2}$ (via elliptic integrals) onto a round annulus $A$ with inner radius 1 and outer radius $R$, here $R$ depends only on $\sigma$. Furthermore, we can choose $\phi_{2}$ such that $\phi_{2}([-i \sqrt{\sigma}, i \sqrt{\sigma}])=\partial \mathbb{D}^{2}$, and $\phi_{2}$ is symmetric with respect to both the $x$ and $y$-axis, in particular, $\phi_{2}(i \sqrt{\sigma})=i$. Finally, let $\phi_{3}(z)=z-1 / z$. This mapping maps $A$ conformally onto the ellipse $E_{1}$ with semi-axes $R-1 / R$ and $R+1 / R$ and foci $\pm i$, with a slit along the imaginary axis from $-2 i$ to $2 i$.

Define three more maps $\tau_{1}, \tau_{2}, \tau_{3}$, where $\tau_{1}=\phi_{1}, \tau_{2}=\phi_{2}$, but $\tau_{3}$ is given by $\tau_{3}(z)=z+1 / z$. This mapping maps the annulus $A$ onto a different ellipse: its semi axes are $R+1 / R$ and $R-1 / R$ and its foci are the points \pm 2 .

The composition $\phi=\phi_{3} \circ \phi_{2} \circ \phi_{1}$ is a conformal mapping from $O \backslash[-\sigma, 0]$ onto the ellipse $E_{1} \backslash[-2 i, 2 i]$, and extends to the slits from 0 to $-\sigma$ in $O$ so that $\phi(0)=0$, $\phi((-1,0))=(-i(R+1 / R), i(R+1 / R))$, and $\phi((0,1))=(-(R-1 / R), R-1 / R)$. Here, $\phi((-1,0))$ stands for the image under $\phi$ of the two lines on the double cover $O$ of the unit disk above the negative real axis in $\mathbb{D}^{2}$ (and similarly interpreted for $\phi((0,1)))$. 
Similarly, the composition $\tau=\tau_{3} \circ \tau_{2} \circ \tau_{1}$ is a conformal mapping from $O \backslash[-\sigma, 0]$ onto the ellipse $E_{2} \backslash[-2,2]$. Note that $\tau$ can be extended to the slits, and the extension has a branch point at $-\sigma$, i.e. $\tau$ extends to the double cover $\tilde{O}$ of the unit disk, branched at $-\sigma$.

The mapping $\Phi$ is finally obtained by mapping $E_{1}$ onto $E_{2}$ with the affine map

$$
T(u+i v)=u \frac{R+\frac{1}{R}}{R-\frac{1}{R}}+i v \frac{R-\frac{1}{R}}{R+\frac{1}{R}} .
$$

Then $\tau_{1}^{-1} \tau_{2}^{-1} \tau_{3}^{-1} T \phi_{3} \phi_{2} \phi_{1}$ is a quasiconformal mapping from $O$ (branched at 0 ) onto $\tilde{O}$ (branched at $-\sigma$ ) that agrees on both sheets and thus descends to a quasiconformal mapping $\Phi: \mathbb{D}^{2} \rightarrow \mathbb{D}^{2}$ that maps 0 to $-\sigma$. The only place where $\Phi$ picks up quasiconformal dilatation is the mapping $T$ that sends the ellipse $E_{1}$ onto the ellipse $E_{2}$.

We will now analyze the direction of maximal distortion for points $z \in(-1,0)$ and points $w \in(0,1)$. The points $z \in(-1,0)$ in the unit disk correspond to points on the imaginary axis in $E_{1}$, and points $w \in(0,1)$ correspond to points on the real axis in $E_{1}$. Thus infinitesimal circles centered at points $z \in(-1,0)$ get mapped under $\Phi$ onto infinitesimal ellipses centered at points on $(-1,-\sigma)$ with major axis orthogonal to $\mathbb{R}$. On the other hand, infinitesimal circles centered at points $w \in(0,1)$ get mapped under $\Phi$ onto infinitesimal ellipses centered at points on $(-\sigma, 1)$ with major axis along $\mathbb{R}$.

Let now $\Gamma$ be a Fuchsian group of the first kind. Then $\Gamma$ contains a hyperbolic element $\gamma$ whose axis $A_{\gamma}$ is arbitrarily close to $(-1,1)$. Since $\Phi$ is a smooth mapping on $\mathbb{D}^{2} \backslash\{0\}$, the line field of $\Phi$ varies continuously in $\mathbb{D}^{2} \backslash\{0\}$. Hence, on a segment of the axis $A_{\gamma}$ the line field of $\Phi$ is almost vertical, whereas on another segment of $A_{\gamma}$ the line field of $\Phi$ is almost horizontal. But some power of $\gamma$ (or $\gamma^{-1}$ ) maps points from the vertical segment into the horizontal segment, but the image of the vertical line field under $D \gamma$ is not the horizontal line field, and thus $\Phi \circ \gamma \neq \gamma \circ \Phi$. Since $\Phi$ is the identity on $\partial \mathbb{D}^{2}$, the only possibility for $\Phi$ to be compatible with $\Gamma$ would be to satisfy $\Phi \circ \gamma=\gamma \circ \Phi$ for all $\gamma \in \Gamma$. Thus no Fuchsian group of the first kind is compatible with $\Phi$, and so $\Phi$ is not the lift of any quasiconformal mapping on any surface whose underlying Fuchsian group is of the first kind. 
In particular, we have shown that our original mapping $\tilde{f}$ (the lift of $f: S \rightarrow S$ to the unit disk) cannot agree with the mapping $\Phi$. Since $\Phi$ is unique with minimal distortion via Teichmüller's result, we conclude that $K(\tilde{f})>K(\Phi)$ and this proves the theorem.

Remark. A fully general $n$-dimensional analogue $(n \geq 3)$ to the Teichmüller extremal result is not known. However, under certain restrictive assumptions a solution has been developed ([1]); it is shown that an extremal map is a rotation of the 2-dimensional extremal mapping.

\section{Amenability and passage of $K_{a u t}$ to a cover}

Let $X$ be a closed hyperbolic surface, and $Y$ be a regular cover of $X$. Since a homeomorphism of $X$ may not lift equivariantly to a homeomorphism of $Y$, it is hard to relate the quasiconformal homogeneities of $X$ and $Y$. However, if we assume that $X$ has trivial conformal automorphism group, then we can cite Lemma 1.4, and thus we quickly observe that $K(Y) \leq K(X)$. If $Y$ is a nonamenable regular cover then we can promote this inequality to a strict inequality.

In order to introduce amenability we first must fix some notation. Let $G$ be a graph and $V$ be any set of vertices in $G$. The boundary $\partial V$ of $V$ is the set of vertices in $G-V$ that are a distance one from $V$ (that is, there is an edge in $G$ that connects a vertex in $\partial V$ to a vertex in $V$.) Define the expansion $\gamma$ of $G$ to be the infimum of $\frac{|\partial V|}{|V|}$ as $V$ varies over all finite vertex subsets of $G$. The group $G$ is said to be amenable if $\gamma=0$, and if $G$ is not amenable it is non-amenable. Let $Y$ be a regular cover of $X$, which we will denote by $\pi: Y \rightarrow X$. We say that $Y$ is an amenable regular cover if the covering group is amenable (here the graph in question is a graph of the group; the property of being amenable persists to every graph of a group.) If the covering group is non-amenable we say that $Y$ is a non-amenable cover of $X$. See McMullen [10] for a more general presentation of amenable and non-amenable covers of Riemann surfaces.

The following is our second primary result in this note.

Theorem 3.1. Let $X$ be a closed Riemann surface with Aut $(X)=\{i d\}$. Let $Y$ be a regular, non-amenable cover of $X$. Then

$$
K_{\text {aut }}(Y)<K_{\text {aut }}(X) .
$$


In order to prove this theorem we first show a local version:

Lemma 3.2. Suppose that $X$ is a closed Riemann surface having trivial automorphism group, and $Y$ be a regular non-amenable cover of $X$. Let $x_{1}, x_{2}$ be two distinct points in $X$ and let $y_{1} \in \pi^{-1}\left(x_{1}\right), y_{2} \in \pi^{-1}\left(x_{2}\right)$ be preimages of $x_{1}, x_{2}$ under the covering map $\pi$. Then

$$
K_{\text {aut }}\left(y_{1}, y_{2}\right)<K_{\text {aut }}\left(x_{1}, x_{2}\right)
$$

Proof. Let $x_{1}, x_{2} \in X$ be two distinct points. Let $f$ be a quasiconformal mapping of $X$ that is homotopic to the identity, that maps $x_{1}$ to $x_{2}$ and that satisfies $K(f)=K_{\text {aut }}\left(x_{1}, x_{2}\right)$. Let $X_{1}$ be the punctured surface $X \backslash\left\{x_{1}\right\}$, and let $X_{2}$ the surface $X \backslash\left\{x_{2}\right\}$. Then $f_{\mid X_{1}}: X_{1} \rightarrow X_{2}$ is extremal in its homotopy class since otherwise there would be a mapping homotopic to $f_{\mid X_{1}}$ on $X_{1}$ with smaller dilatation. But filling in the punctures would then yield a quasiconformal mapping of $X$ that maps $x_{1}$ to $x_{2}$, which is homotopic to the identity and has dilatation smaller than $K_{\text {aut }}\left(x_{1}, x_{2}\right)$ which is a contradiction.

Now let $Y_{1}=Y \backslash \pi^{-1}\left(x_{1}\right)$ and $Y_{2}=Y \backslash \pi^{-1}\left(x_{2}\right)$. Then $f_{\mid X_{1}}$ lifts to a mapping $\tilde{f}$ from $Y_{1}$ to $Y_{2}$ (since $f$ itself is homotopic to the identity it lifts to a quasiconformal homeomorphism of $Y$ to itself; the lift can be chosen to be homotopic to the identity on $Y$ ). Since by assumption $Y$ is a non-amenable regular cover of $X$ we can conclude that $Y_{1}$ is a non-amenable regular cover of $X_{1}$ (in fact, the covering groups $\Pi_{1}(X) / \Pi_{1}(Y)$ and $\Pi_{1}\left(X_{1}\right) / \Pi_{1}\left(Y_{1}\right)$ are identical). Hence because $X_{1}$ is of finite type and $Y_{1}$ is a non-amenable cover of $X_{1}$, McMullen's result [10, Corollary 1.2] implies that $\tilde{f}: Y_{1} \rightarrow Y_{2}$ is not extremal in its homotopy class. Hence there exists a quasiconformal homeomorphism $g: Y_{1} \rightarrow Y_{2}$ which is homotopic (in $Y_{1}$ ) to $\tilde{f}$ such that $K(g)<K(\tilde{f})$. Now $g$ can be extended to a quasiconformal mapping (again denoted $g$ ) of $Y$ to itself which maps the set of punctures $\pi^{-1}\left(x_{1}\right)$ to the set of punctures $\pi^{-1}\left(y_{1}\right)$ in the same way that $\tilde{f}$ did. Since $\tilde{f}$ (when extended to all of $Y$ ) is homotopic to the identity by choice of the lift, we see that the extended $g$ is homotopic to the identity on $Y$ as well and $K(g)<K(\tilde{f})$. Furthermore, $g$ maps $y_{1}$ to some point $\alpha\left(y_{2}\right)$ for some $\alpha \in \Pi_{1}(X) / \Pi_{1}(Y)=\operatorname{Aut}(Y)$. Then $\alpha^{-1} \circ g$ maps $y_{1}$ to $y_{2}$, and thus we have that

$$
K_{\text {aut }}\left(y_{1}, y_{2}\right) \leq K\left(\alpha^{-1} \circ g\right)<K(\tilde{f})=K_{\text {aut }}\left(x_{1}, x_{2}\right),
$$

which completes the proof. 
Proof of Theorem 3.1. We will show more generally that if $X$ is an arbitrary compact hyperbolic surface and $Y$ is a regular cover of $X$ with covering map $\pi: Y \rightarrow X$ such that $K_{\text {aut }}^{Y}\left(y_{1}, y_{2}\right)<K_{\text {aut }}^{X}\left(x_{1}, x_{2}\right)$ for any two distinct $x_{1}, x_{2} \in X$ and any preimages $y_{1} \in \pi^{-1}\left(x_{1}\right), y_{2} \in \pi^{-1}\left(x_{2}\right)$, then $K_{\text {aut }}(Y)<K_{\text {aut }}(X)$.

To do so, pick $a, b \in Y$ such that $K_{\text {aut }}^{Y}(a, b)=K_{a u t}(Y)$. Such two points exist (i.e. $K_{a u t}(Y)$ is attained) since $Y$ is a regular cover of the compact surface $X$, and hence there exists a compact fundamental domain for the action of $Y$ 's automorphism group on $Y$. The existence of $a$ and $b$ now follows from a simple compactness / normal family argument. But by assumption we have that $K_{\text {aut }}^{Y}(a, b)<K_{\text {aut }}^{X}(\pi(a), \pi(b))$, and this implies that

$$
K_{\text {aut }}(Y)=K_{\text {aut }}^{Y}(a, b)<K_{\text {aut }}^{X}(\pi(a), \pi(b)) \leq K_{\text {aut }}(X),
$$

completing the proof.

We finish this note with an immediate application of Theorem 3.1; recall that a free group having two or more generators is non-amenable. Recall also that the Retrosection theorem provides for the existence of (necessarily non-amenable) planar covers of any closed hyperbolic surface (see [3] for a discussion of the Retrosection theorem.)

Corollary 3.3. Let $X$ be a closed hyperbolic surface with trivial automorphism group. Then any planar hyperbolic domain $\Omega$ that is obtained from $X$ by the Retrosection Theorem has the property that $K_{\text {aut }}(\Omega)<K_{\text {aut }}(X)$.

It would be interesting to know if, for any fixed genus $g \geq 3$, there is a uniform bound $c_{g}<1$ (over all closed conformal surfaces $X$ of genus $g$ having trivial automorphism groups) so that $K_{\text {aut }}(\Omega) / K_{\text {aut }}(X) \leq c_{g}$ for any domain $\Omega$ obtained from a retrosection of $X$.

\section{REFERENCES}

1. G.D. Anderson and M.K. Vamanamurthy, An extremal displacement mapping in $n$-space, Complex analysis Joensuu 1978 (Proc. Colloq., Univ. Joensuu, Joensuu, 1978), pp. 1-9, Lecture Notes in Math., 747, Springer, Berlin, 1979.

2. A. Beardon, The geometry of discrete groups, Springer-Verlag, New York, 1995.

3. L. Bers, Automorphic forms for Schottky groups, Advances in Mathematics 16 (1974), $332-361$. 
4. P. Bonfert-Taylor, R. D. Canary, G. Martin, and E. C. Taylor, Quasiconformal homogeneity of hyperbolic manifolds, Math. Ann. 331 (2005), 281-295.

5. P. Bonfert-Taylor, M. Bridgeman, R. D. Canary, and E. C. Taylor, Hyperbolic surfaces with fixed-point full automorphisms, Math. Proc. Cambridge Philos. Soc., (2007) 143, 71-84.

6. C. Earle and I. Kra, On holomorphic mappings between Teichmüller spaces, in Contributions to analysis, L. Ahlfors et al (eds.), Academic Press, New York, 1974, 107-124.

7. F. W. Gehring and B. Palka, Quasiconformally homogeneous domains, J. Analyse Math. 30 (1976), 172-199.

8. P. MacManus, R. Näkki, and B. Palka, Quasiconformally homogeneous compacta in the complex plane, Michigan Math. J. 45 (1998), 227-241.

9. P. MacManus, R. Näkki, and B. Palka, Quasiconformally bi-homogeneous compacta in the complex plane, Proc. London Math. Soc. 78 (1999), 215-240.

10. C. McMullen, Amenability, Poincaré series, and quasiconformal maps, Invent. Math. 97 (1989), no. 1, 95-127.

11. G. P. Scott, Subgroups of surface groups are almost geometric, J. London Math. Soc. 17 (1978), 555-565. See also ibid Correction: J. London Math. Soc. 32 (1985), 217-220.

12. J. Schneider, Zum Isodiametrischen Problem im Hyperbolischen Raum, Manuscripta Math. 60 (1988), 437-461.

13. O. Teichmüller, Ein Verschiebungssatz der quasikonformen Abbildung, Deutsche Math. 7 (1944), 336-343.

Petra Bonfert-Taylor

Wesleyan University,

Middletown, Connecticut

USA

E-mail: pbonfert@wesleyan.edu

Gaven Martin

Massey University,

Albany, Auckland

New Zealand

E-mail: G.J.Martin@massey.ac.nz

\author{
Alan W. Reid \\ University of Texas, \\ Austin, Texas
}


USA

E-mail: areid@math.utexas.edu

Edward C. Taylor

Wesleyan University,

Middletown, Connecticut

USA

E-mail: ectaylor@wesleyan.edu 